\title{
Prüfungsrecht: Das Problem der Prüfungsdelegation
}

\author{
Martin Tegelkamp/Steven Kensy*
}

A. Einleitung ......................... 482

B. Zulässigkeit der Vorkorrektur ........ 483

I. Korrekturmodelle ................ 483

II. Zulässigkeit der nachvollziehenden und mittelbaren Korrektur.......... 484

1. Die Person des Prüfers.......... 484

2. Die Berufung zum Prüfer........ 490
3. Normative Grundlagen der Vorkorrektur und ihre Flexion in der

Rechtsprechung ................ 491

C. Korrekturpraxis .................... 494

D. Reformvorschläge .................. 495

E. Zusammenfassung .................. 496

Im Zentrum der kontrovers diskutierten Hochschulreform steht die Verbesserung der Lehrsituation an deutschen Universitäten. Hochschulausbildung indes ist ohne Leistungskontrolle nicht denkbar. Die folgende Darstellung wendet sich der Frage nach möglichen Reformen de lege ferenda im Zusammenhang mit der Prüfungspraxis Juristischer Fakultäten zu. Den Gegenstand bildet die Vorkorrektur in Ausgestaltung und Zulässigkeit. ${ }^{1}$

\section{A. Einleitung}

Anspruch und Wirklichkeit klaffen in vielen Bereichen des öffentlichen und privaten Lebens auseinander. Dass sich dieses Phänomen einmal mehr gerade bei der Abnahme von Prüfungen im Studium der Rechtswissenschaften quer durch eben jene Republik findet, die sich mit Stolz ihrer nunmehr schon über 60 Jahre fundierten Rechtsstaatlichkeit besinnt, verwundert; wirft aber die Frage auf, inwieweit Rechtssetzung ohne Rücksicht auf die praktischen Bedürfnisse funktionieren kann.

An den heutigen Massenuniversitäten wird der stetig steigenden Studierendenzahl von Seiten der Prüfenden praktisch derart begegnet, dass ihre persönliche Mitwirkung bei der Leistungskontrolle auf ein möglichst geringes Maß reduziert wird. Dies geschieht im Wesentlichen mittels einer „Vorkorrektur“ schriftlicher Arbeiten durch prüferverschiedene Personen. Die nachstehende Untersuchung nimmt dieses Vorgehen zum Anlass, die derzeitigen Prüfungsverfahren an Juristischen Fakultäten genauer zu betrachten.

Zunächst werden im Folgenden Gegenstand und Zulässigkeit der verschiedenen Formen der Vorkorrektur (mittelbare und nachvollziehende) behandelt (B.), im Anschluss daran werden die rechtlichen Anforderungen an ihrer praktischen Umsetzung gemessen (C.). Die dabei zu Tage tretende Inkongruenz von Anspruch und Prü-

* Martin Tegelkamp, LL.M. ist Wissenschaftlicher Mitarbeiter am Institut für Rechtsgeschichte, Germanistische und Kanonistische Abteilung der Rechtswissenschaftlichen Fakultät der Westfälischen Wilhelms-Universität Münster, Steven Kensy ist Wissenschaftlicher Mitarbeiter am selben Institut und Lehrkraft für besondere Aufgaben an der Rechtswissenschaftlichen Fakultät der Westfälischen Wilhelms-Universität Münster.

1 Zum Problemaufriss schon S. Kensy/M. Tegelkamp, Von Nöten und Zwängen juristischer Fakultäten im Umgang mit dem Prüfungsrecht, JuS 2010, XXXII-XXXVI. 
fungswirklichkeit mündet schließlich in einen Vorschlag zur Änderung des Verfahrens, bei dessen Verwirklichung ein rechtmäßiges Prüfungsverhalten der beteiligten Akteure gewährleistet wäre (D.). Die Untersuchung schließt mit einer in die Praxis schauenden Zusammenfassung (E.).

\section{B. Zulässigkeit der Vorkorrektur}

Um der Frage nach der Zulässigkeit einer Vorkorrektur sinnvoll nachgehen zu können, bedarf es zunächst einer Begriffsbestimmung, der sich eine Definition des Prüfers anzuschließen hat, um dessen gegenständliche Abgrenzung zu einem „Vorkorrektor“ zu ermöglichen. Sodann kann nach den normativen Grundlagen und den Anforderungen, welche die Rechtsprechung an Vorkorrekturen stellt, gefragt werden. Die Untersuchung beschränkt sich hierbei aus der Natur der Sache auf die Bewertung schriftlicher Leistungen und setzt den Schwerpunkt bezüglich der Berücksichtigung von Landesrecht bei Nordrhein-Westfalen; die Regelungen im Bundesgebiet ähneln sich aber tendenziell.

\section{Korrekturmodelle}

Neben einer an deutschen Universitäten nur für den Schwerpunktbereich explizit vorgesehenen „höchstpersönlichen“ Korrektur schriftlicher Prüfungsleistungen durch den Prüfer, ${ }^{2}$ in deren Rahmen jede Mitwirkung Dritter bei der Bewertung ausgeschlossen ist, ${ }^{3}$ existieren traditionell zwei Modelle, die „nachvollziehende “ und die „mittelbare“ Korrektur. ${ }^{4}$

Die „nachvollziehende“ Korrektur erfolgt in der Weise, dass dem Prüfer zugeordnete Korrekturassistenten die Klausuren (zumeist anhand einer Lösungsskizze) vorkorrigieren, woraufhin der Prüfer diese vorkorrigierten Arbeiten persönlich liest, um sich selbstständig ein Urteil über die Bewertung zu bilden.

Das zweite - sog. mittelbare oder auch stellvertretende - Modell verlagert die persönliche Tätigkeit des Prüfers vollständig in das Vorfeld der eigentlichen Korrektur. Es handelt sich um eine mittels Lösungsskizze angeleitete Korrektur durch weisungsabhängige und dem Prüfer fachlich unterlegene Dritte, wobei sich eine etwaige persönliche Kenntnisnahme der Einzelleistung durch die zum Prüfer bestellte Person auf Stichproben oder eine Nachkorrektur auf Gegenvorstellung beschränkt.

2 Vgl. etwa $\$ 24$ Abs. 3 PO HU Berlin (Schwerpunktbereichsklausur) bzw. für Studienbeginn ab dem WS 2008/09 \$ 6 Abs. 3; $\$ 37$ Abs. 2 S. 1 PO Bremen (Abschlussarbeit); $\$ 12$ Abs. 5 SPO Hamburg (Aufsichtsarbeit); $\mathbb{\$} 15$ Abs. 2 SPO Heidelberg (Schwerpunktbereichsleistungen); $\mathbb{} 15$ Abs. 1 SPO Konstanz (Aufsichtsarbeit).

3 N. Niehues/E. Fischer, Prüfungsrecht, München 2010, Rn. 320.

4 Vgl. D. Heckmann/B. Vogler, Bewertungsgrundsätze für die „studienbegleitende Zwischenprüfung“, JZ 1998, S. 637 (638 f.). 


\section{Zulässigkeit der nachvollziehenden und mittelbaren Korrektur}

In beiden Varianten der Korrektur stellt sich die Problematik, inwiefern die verantwortliche Person (in aller Regel der Leiter einer Lehrveranstaltung) sachlich noch der Prüfer ist, wenn die Korrektur an Dritte delegiert wird, und inwieweit die Bewertung der Leistung dann noch vom fachwissenschaftlichen Urteil des verantwortlich Unterzeichnenden abhängt. ${ }^{5}$

\section{Die Person des Prüfers}

Ist für die Sonderdelikte im Strafrecht so prägnant wie treffend formuliert, „Täter kann nur sein, wer Täter sein kann“, mag im Prüfungsrecht als Ausgangspunkt für die Person des Prüfers gelten, „Prüfer kann nur sein, wer Prüfer sein kann“. Damit sind zunächst die formalen und fachlichen Qualifikationen angesprochen. Neben der „allgemeinen“ Qualifikation, etwa der Unbefangenheit und Sachlichkeit eines Prüfers, welche nicht im Fokus dieser Betrachtung stehen soll, ist vorderste Anforderung die Fähigkeit zur Ermittlung und sachgerechten Beurteilung des Wertes einer erbrachten Leistung (fachliche Qualifikation). Normativ wird diesem verfassungsrechtlich aus dem Gebot der Chancengleichheit (Art. 3 Abs. 1 i.V.m. Art. 12 Abs. 1 GG) hergeleiteten Erfordernis dadurch Rechnung getragen, dass Prüfungsleistungen nach $\mathbb{1 5}$ Abs. 4 HRG n.F. und den einschlägigen Regelungen der Bundesländer ${ }^{6}$ nur von Personen bewertet werden dürfen, die mindestens die „durch die Prüfung festzustellende" oder eine "gleichwertige "Qualifikation besitzen. Vielfach stellen die Länder überdies qualifiziertere Anforderungen und verlangen für die Abnahme von Hochschulprüfungen einen Hochschullehrerstatus, die Lehrtätigkeit des Prüfenden oder wenigstens eine entsprechende Befugnis; gelegentlich sind daneben in der beruflichen Praxis und Ausbildung erfahrene Personen als Prüfer ausdrücklich zugelassen (formale Qualifikation). ${ }^{7}$

Soweit die zitierten landesrechtlichen Regelungen ihrem Wortlaut nach maßgeblich auf die eigenverantwortliche Wahrnehmung von Lehraufgaben abstellen, folgt die

5 Vgl. J. Pietzcker, Verfassungsrechtliche Anforderungen an die Ausgestaltung von Prüfungen, Diss. Berlin 1975, S. $117 \mathrm{f}$

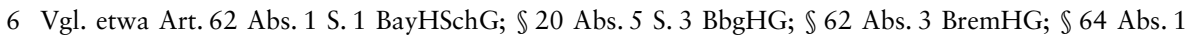
HmbHG; $\mathbb{} 18$ Abs. 2 S. 2 HHG; $\mathbb{\int} 36$ Abs. 4 S. 3 LHG M-V; $\mathbb{S} 65$ Abs. 1 S. 2 NRW HG; $\int 58$ Abs. 3

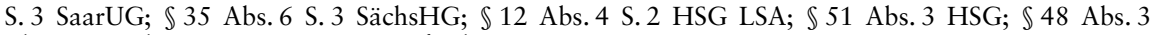
ThürHG; teilweise mit strengeren Anforderungen.

7 Vgl. etwa Art. 62 Abs. 1 Nr. 2 BayHSchG; $\$ 20$ Abs. 5 S. 1 BbgHG, allerdings mit der Maßgabe, dass Hochschulprüfungen nur von Personen abgenommen werden sollen, die Lehraufgaben wahrnehmen; $\$ 62$ Abs. 3 BremHG a.F., jetzt in $\$ 62$ Abs. 3 S. 1 mit speziellem Fokus auf eine haupt- oder nebenbe-

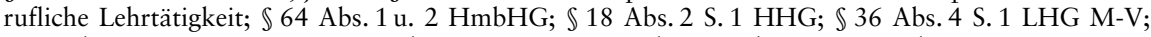

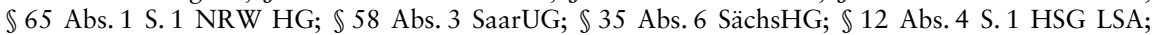
$\$ 48$ Abs. 2 ThürHG; wobei es sich teilweise um Sollvorschriften handelt. 
Prüfungsbefugnis dem allgemein anerkannten Grundsatz ${ }^{8}$ „Wer lehrt, prüft“. Dies greift jedoch nach Auffassung der Rechtsprechung zu kurz. Fehlte im Regierungsentwurf zu $\$ 15$ Abs. 4 HRG a.F. noch eine statusabhängige Mindestqualifikation, so wurde diese im Nachgang zu einer Entscheidung des Bundesverwaltungsgerichts eingefügt, in welcher das Gericht die Beurteilung von Prüfungsleistungen bei Promotionen und Habilitationen durch Personen, die nicht mindestens eine vergleichbare Qualifikation besitzen, mit der Wertentscheidung des Art. 5 Abs. 3 GG und der eingeschränkten gerichtlichen Nachprüfbarkeit von Prüfungsentscheidungen für unvereinbar erklärt hat. ${ }^{9}$ Die Forderung des Hochschulrahmengesetzes der „gleichwertigen" oder "durch die Prüfung festzustellenden Qualifikation" stellt demnach eine statusabhängige Begrenzung der aufgrund ihrer Lehrtätigkeit grundsätzlich prüfungsbefugten Personen dar. Bei der erforderlichen formalen Qualifikation handelt es sich allerdings lediglich um ein Mittel zur Sicherung des fachlichen Standards und nicht um ein aliud im Sinne einer zusätzlichen oder weiterreichenden Qualifikation. Denn die fachliche Qualifikation eines Prüfers, eine Leistung sachgerecht beurteilen zu können, kann praktisch nur über seine formale Qualifikation sichergestellt werden; diese fachliche Qualifikation ist aber aus verfassungsrechtlicher Sicht allein maßgebend.

Eine differenziertere gesetzliche oder hochschulrechtliche Regelung zur Qualifikation des Prüfers existiert nicht. Auch besteht verfassungsrechtlich kein zwingendes Bedürfnis, dass der Prüfer gerade auf dem Gebiet, aus dem die Prüfungsaufgabe stammt, tätig oder besonders spezialisiert ist. ${ }^{10}$ Zwar wird in aller Regel die Sachkompetenz eines Prüfers hinsichtlich der Beurteilung einer Leistung am deutlichsten erkennbar sein, wenn sich dieser selbst der zu bewertenden Leistungskontrolle erfolgreich unterzogen hat. Wie aber das Kompetenzzugeständnis des Hochschulrahmengesetzes und der einschlägigen landesrechtlichen Regelungen an „gleichwertig“ qualifizierte Personen in Bezug auf ihre Prüfertätigkeit bereits zeigt, existiert kein ungeschriebener (verfassungs- oder bundesrechtlicher) Prüfungsgrundsatz, dass ein Prüfer gerade die abzunehmende Leistungskontrolle stets selbst erfolgreich abgelegt haben müsste. ${ }^{11}$

8 So der ausdrückliche Hinweis des Gesetzgebers im Gesetzesentwurf der Bundesregierung zum Hochschulrahmengesetz zu $\$ 16$ Abs. 4, welcher im Wesentlichen inhaltsgleich mit der bis zum 24.8.1998 geltenden Fassung des $\$ 15$ HRG den zur Abnahme von Prüfungen befugten Personenkreis enumerativ festlegte, vgl. BT-Drs. 7/1328, S. 47; vgl. dazu M. Weber, in: D. Leuze/V. Epping (Hrsg.), Kommentar zum Hochschulgesetz Nordrhein-Westfalen, Loseblatt-Sammlung, Stand: November 2007, $\$ 65$ Rn. 2.

9 BVerwG, Urt. 22.2.1974 - VII C 9/71 = BVerwGE 45, 39 ff.; vgl. H.-W. Waldeyer, in: K. Hailbronner/ M.-E. Geis, Hochschulrecht in Bund und Ländern, vorher Kommentar zum Hochschulrahmengesetz, Mai 1999, $\mathbb{1} 15$ Rn. 41.

10 BVerwG, Beschl. v. 20.8.1997 - 6 B 25/97; Beschl. v. 20.11.1995 - 6 B 66/95; Niehues, Prüfungsrecht (Fn. 3), Rn. 164.

11 Niehues/Fischer, Prüfungsrecht (Fn. 3), Rn. 306; BVerwG, Beschl. v. 27.3.1992 - 6 B 6/92. 
Bei strikter Handhabung eines solchen Erfordernisses wären die Universitäten, unter Aufgabe ihrer - in Nordrhein-Westfalen seit Inkrafttreten des Hochschulfreiheitsgesetzes 2007 ausdrücklich verbrieften - Autonomie, sogar gezwungen, ihre jeweiligen Studienordnungen einander anzugleichen, weil andernfalls nicht gewährleistet werden könnte, dass sich überhaupt Prüfer in ausreichender Zahl finden lassen, die genau diese Art von Prüfung selbst abgelegt haben, welche es in einem bestimmten Fall abzunehmen gilt. Den Faden zu Ende gesponnen, bedeutete ein solches Postulat, dass weder Studieninhalte verändert, noch neue Studieninhalte oder -gänge überhaupt entwickelt und etabliert werden könnten. Dass man dann von Freiheit nicht mehr sprechen könnte, der amtliche Titel eines „Hochschulfreiheitsgesetzes“ vielmehr bloßer Alogismus wäre, lässt offensichtlich werden, dass der moderne Gesetzgeber die Hochschulen formal soweit nicht gängeln wollte.

Das eigentliche Problem in Bezug auf die Person des Prüfers liegt also in der schon angesprochenen Verknüpfung von Lehre und Prüfungsberechtigung. Wenn $\$ 65$ Abs. 1 S. 1 HG NRW bestimmt, zur Abnahme von Prüfungen seien von den in beruflicher Praxis und Ausbildung erfahrenen Personen ${ }^{12}$ einmal abgesehen, die an der Hochschule Lehrenden befugt, ist zum einen fraglich, ob es sich hierbei um ein Exklusivitätsverhältnis in dem Sinne handelt, dass ausschließlich Lehrende prüfen dürfen und es den Universitäten verwehrt ist, andere Personen, wie etwa wissenschaftliche Hilfskräfte oder ausländische Gastprofessoren mit Prüfungsabnahmen zu betrauen. Zum anderen, und dies scheint die brisantere Frage zu sein, bleibt offen, was das Gesetz unter Lehre versteht.

Was das Exklusivitätsverhältnis anlangt, kann eine Tätigkeit in der Hochschullehre als zwingende Voraussetzung für die Prüfungsbefugnis dem Wortlaut des \65 Abs. 1 S. 1 HG NRW zwar nicht unmittelbar entnommen werden. Eine systematische Auslegung der Vorschrift veranlasst jedoch dazu, eine noch näher zu beleuchtende Lehrtätigkeit zu fordern. Gerade weil bestimmte Hochschulexterne aus dem Bereich der Praxis über den Kreis der Lehrenden hinaus zur Prüfungsabnahme berechtigt werden, kann man nicht davon ausgehen, dass der Gesetzgeber den „freien " Hochschulen anheimstellen wollte, sonstige Dritte zu Prüfern zu bestellen. Dieser noch recht einleuchtende Befund, der willkürlichen Prüferbestellung den Riegel vorzuschieben und den Universitäten trotzdem im Sinne einer landesrechtlichen Deregulierung die Möglichkeit einzuräumen, Engpässen verfügbarer Lehrkräfte zu begegnen und eine Interaktion mit der Praxis zu fördern, leitet über zu der weichenstellenden zweiten Frage, welche Personen „die an der Hochschule Lehrenden sind“. 
Was Lehre im Sinne des $\$ 65$ Abs. 1 S. 1 HG NRW meint, verrät das Gesetz in seinem Sechsten Abschnitt mit der amtlichen Überschrift „Lehre, Studium und Prüfungen“ nicht. Überwiegend wird die Konkretisierung des Lehrbegriffes deshalb anhand der Kategorien freie Lehre und weisungsabhängige Lehre versucht. ${ }^{13}$ Von der fehlenden Differenzierung im Sechsten Abschnitt einmal abgesehen, lässt sich eine derartige Systematisierung jedenfalls dann nachvollziehen, wenn auch der Vierte Abschnitt über das Hochschulpersonal bei der Auslegung des $\$ 65$ Abs. 1 S. 1 HG NRW berücksichtigt wird. Dort stellt das Gesetz in $\mathbb{S} 44$ Abs. 1, 2 S. 1 HG NRW die weisungsabhängige der weisungsfreien Tätigkeit in $\mathbb{} 44$ Abs. 2 S. 2 HG NRW gegenüber. Da in beiden Fällen von Lehre die Rede ist, anerkennt das Gesetz, dass es sich hierbei um den Obergriff handelt.

Für die Auslegung des Lehrbegriffs in $\$ 65$ Abs. 1 S. 1 HG NRW kommt es daher nun maßgeblich darauf an, ob die Vorschrift nur einen dieser Lehrbegriffe in Bezug nimmt. Sollte die Bestimmung des $\ 65$ Abs. 1 S. 1 HG NRW nämlich auch die weisungsabhängige Lehre erfassen, wäre es den Universitäten unbenommen, auch Wissenschaftliche Mitarbeiter mit der Prüfungsabnahme zu betrauen, soweit sie mit entsprechenden Lehraufgaben wie etwa der Leitung einer vorlesungsbegleitenden Arbeitsgemeinschaft befasst sind. Aus normhistorischer Perspektive betrachtet, spricht vor allem die Neufassung des $\$ 65$ Abs. 1 S. 1 HG NRW gegenüber der bis zum 31.12.2006 geltenden Fassung des $\mathbb{S} 95$ Abs. 1 S. 1 HG NRW, welcher den zur Abnahme von Hochschulprüfungen befugten Personenkreis noch enumerativ festlegte, dafür, auch Wissenschaftliche Mitarbeiter als „, an der Hochschule Lehrende“ zu begreifen. So fand sich in $\mathbb{\$} 95$ Abs. 1 S. 1 HG NRW a.F. noch die ausdrückliche Einschränkung, dass wissenschaftliche Mitarbeiter zur Abnahme von Hochschulprüfungen berechtigt seien, „soweit sie Aufgaben nach \59 I 4 HG NRW a.F. wabrnehmen". Anders als das geltende Recht enthielt die alte Regelung zum prüfungsbefugten Personenkreis damit einen expliziten Verweis aus dem damaligen Siebten Abschnitt „Lehre, Studium und Prüfungen“ auf die weisungsfreie Lehre als Voraussetzung der Prüfungsbefugnis. Klarstellend heißt es im Gesetzesentwurf der Landesregierung zu $\mathbb{} 95 \mathrm{HG}$ NRW a.F.:

„Wissenschaftliche Assistentinnen und Assistenten sowie wissenschaftliche Mitarbeiterinnen und Mitarbeiter an den Universitäten sind zur selbstständigen Abnahme von Prüfungen nur soweit befugt, als sie nach $\$ 56$ I Satz 5 und \59 II Satz 2 selbstständig lebren dürfen. "14

Fehlt in der Neufassung des Sechsten Abschnitts über Lehre und Prüfungswesen nunmehr ein differenzierender Verweis auf die Natur der Lehrtätigkeit als frei oder 
gebunden, zeigt sich ein zwischen abhängiger und freier Lehre nicht unterscheidender objektivierter Wille des Gesetzgebers auch im Regierungsentwurf zum Hochschulfreiheitsgesetz bestätigt. Zu $\$ 65$ HG NRW liest man dort:

„Die Vorschrift enthält in ihren Absätzen 1 und 2 die redaktionell angepassten Regelungen des $\$ 95$ Abs. 1 und 2 HG a.F. und führt dessen Gehalt zugleich auf ein Regulierungsniveau zurück, welches einer selbstständigen Körperschaft angemessen ist." 15

Wenn es demnach die Absicht des Gesetzgebers gewesen ist, das landesrechtliche Regulierungsniveau zugunsten hochschulautonomer Regelungen zurückzunehmen, ist die Neufassung in dem Sinne zu verstehen, dass auch diejenigen Mitarbeiter, die mit weisungsabhängigen Lehraufgaben betraut sind, zur Prüfungsabnahme berufen werden können.

Bestärkt wird ein derartiges Verständnis des Lehrbegriffs in $\$ 65$ Abs. 1 S. 1 HG NRW durch die Lehrverpflichtungsverordnung, ${ }^{16}$ die gerade auch für wissenschaftliche Mitarbeiter eine nicht unbeträchtliche Lehrverpflichtung vorsieht, ohne dass hierbei auf eine Weisungsfreiheit Bezug genommen wird. Denn sollte - in diesem Fall nach dem Verständnis des Verordnungsgebers - nur weisungsfreie Tätigkeit Lehre sein, ist die Erfüllung der Stundenvorgaben in aller Regel unmöglich, stellt doch die weisungsfreie Lehre nach der Vorstellung des Gesetzes gerade die besonders zu begründende Ausnahme dar. Ein Blick in die Praxis zeigt dann auch, dass Wissenschaftliche Mitarbeiter ihr Lehrdeputat durch Anleitung von Tutorien und Arbeitsgemeinschaften abgelten.

Besondere Bedeutung für die Frage nach dem Kreis der zur Abnahme von Hochschulprüfungen Berechtigten wird bisweilen der Vorschrift des $\mathbb{3} 35$ Abs. 1 S. 2 HG NRW beigemessen, ${ }^{17}$ wonach es zu den hauptberuflichen Aufgaben eines Hochschullehrers gehört, Prüfungen abzunehmen. Weil Hochschullehrer naturgemäß selbstständig und weisungsfrei lehren und das Gesetz ihnen die Prüfungsberechtigung sogar hauptberuflich zuerkennt, könnte daraus gefolgert werden, dass die Prüfungsbefugnis stets weisungsfreie Lehre als immanenten Bestandteil voraussetzt. Gesetzessystematisch betrachtet stellt sich ein solches Verständnis aber als Zirkelschluss dar. Denn auch $\$ 35$ HG NRW hat ausdrücklich die dienstrechtliche Stellung eines Hochschullehrers zum Gegenstand und trifft daher keine weitergehenden Aussagen über eine dem Lehrbegriff des $\$ 65$ Abs. 1 S. 1 HG NRW immanente Weisungsfreiheit. Wollte man dem Gesetz in $\$ 35$ HG NRW eine derartige Differenzierung mit Geltung auch für die Vorschriften entnehmen, welche die Prüfung zum Gegenstand

15 LT- Drs. 14/2063, S. 168.

16 LVV v. 24.6.2009.

17 Vgl. Weber (Fn. 9), $\$ 65$ Rn. 4. 
haben, fragt sich doch, warum diese Differenzierung nur dort und nicht auch bei der viel größeren Gruppe des sonstigen Hochschulpersonals, einschließlich der Honorarprofessoren und Lehrbeauftragten Eingang gefunden hat. Die Regelung des $\mathbb{3 5}$ HG NRW für eine über die Festlegung der Dienstpflichten hinausgehende Zweiteilung des Lehrbegriffs zu aktivieren, überspannt das Maß an Rechtsschöpfung, welches Auslegung zu gewinnen vermag.

Soweit damit bisher hochschulrechtlich nichts dazu zwingt, der Lehre im Sinne des \65 Abs. 1 S. 1 HG NRW ein Merkmal der Weisungsfreiheit unterzuschieben, bleiben noch verfassungsrechtliche Vorgaben zu erwägen, die bei der Auslegung des Fachrechts zu berücksichtigen sind. Ankerpunkt für die Weisungsfreiheit der Lehre als Voraussetzung für die Prüfertätigkeit kann nur Art. 5 Abs. 3 GG sein. Die hierin verfassungsrechtlich verbriefte Lehrfreiheit wurzelt in der Verbindung von Forschung und Erkenntnisvermittlung und setzt die eigenverantwortliche Erkenntnisgewinnung voraus. Garantiert wird demnach nur die wissenschaftliche Lehre. ${ }^{18}$ Von Art. 5 Abs. 3 GG nicht erfasst sind folglich Lehrveranstaltungen, die von weisungsabhängigen, wissenschaftlich nicht eigenverantwortlich tätigen Personen geleitet werden, etwa Arbeitsgemeinschaften, Tutorien oder auch Vertretungsveranstaltungen eines wissenschaftlichen Mitarbeiters. ${ }^{19}$ Sollte die Prüfungsbefugnis dem schon erwähnten Grundsatz „Wer lehrt, prüft" folgend, voraussetzen, dass sich ein Prüfer bei seiner Tätigkeit auf Art. 5 Abs. 3 GG stützen kann, müsste Lehre im Sinne des $\$ 65$ Abs. 1 S. 1 HG NRW weisungsfrei sein. Die gegenüber der Wissensvermittlung vollkommen unterschiedliche Prüfungssituation muss eine solche Schlussfolgerung aber bereits im Ansatz zurückweisen.

Ohne Rücksicht darauf, dass es bei Prüfungen lediglich darum geht, festzustellen, ob ein Kandidat die erforderlichen Kenntnisse erworben hat, kann die grundrechtliche Freiheitsgarantie zugunsten der eigenverantwortlich Lehrenden nicht zu einer einfachgesetzlich vermittelten Freiheitsbeschränkung der weisungsabhängig Tätigen führen. Sofern die Prüfertätigkeit, eine vermeintliche Lehrakzessorietät einmal unterstellt, überhaupt ein Mindestmaß an abstrakter Eigenverantwortung verlangt, ist diesem Erfordernis durch das in $\$ 44$ Abs. 2 S. 1 HG NRW eingeräumte Recht auf Äußerung der eigenen Lehrmeinung ausreichend Genüge getan.

Dass Eigenverantwortung in diesem Zusammenhang ohnehin nur abstrakt sein kann, ergibt sich bereits aus der Natur der Prüfung als selbstständige Vertretbarkeitskontrolle. Denn würde eine Weisungsbefugnis beispielsweise derart ausgeübt, dass eine bestimmte Lösung als falsch zu werten ist, wäre damit schon der Bewertungsakt vollzogen und zugleich sachlich nicht der Weisungsempfänger der Prüfer, sondern der Weisende. Den Beweis dafür, dass die Prüfungsbefugnis eine verfas-

18 R. Scholz, in: T. Maunz/G. Dürig, Kommentar zum Grundgesetz, 55. Ergl. 2009, Art. 5 Abs. 3 Rn. 104.

19 Scholz (Fn. 19), Art. 5 Abs. 3 Rn. 105. 
sungsrechtliche Lehrfreiheit sachlich gar nicht voraussetzen kann, liefern diejenigen Veranstaltungen, in deren Rahmen es nicht um die Vermittlung von persönlichem Erkenntnisfortschritt, also Wissenschaft, geht. Es gibt eine Vielzahl von Lehrveranstaltungen, die sich auf Reproduktion beschränken. Besonders deutlich zeigt sich dies auch daran, dass es, das Axiom einer notwendigen Lehrfreiheit unterstellt, keinen Prüfer an Fachhochschulen oder Schulen geben könnte. Die Voraussetzungen wissenschaftlicher Lehre sind in diesen Bereichen nämlich nie gegeben. ${ }^{20}$

Damit ist festzustellen, dass auch verfassungsrechtlich kein Bedürfnis besteht, den Kreis der „Lehrenden“ in $\$ 65$ Abs. 1 S. 1 HG NRW durch ein Merkmal der Weisungsfreiheit einzuengen. Wissenschaftliche Mitarbeiter können daher in jedem Fall prüfen, wenn sie ihre vertragliche Lehrverpflichtung erfüllen.

\section{Die Berufung zum Prüfer}

Nun macht die Erfüllung der gesetzlichen Vorgaben allein eine abstrakt prüfungsbefugte Person noch nicht zum Prüfer für die konkrete Leistungskontrolle. Vielmehr bedarf es regelmäßig einer Bestellung durch das zuständige Hochschulorgan. ${ }^{21}$ Dieses ist der nach Maßgabe der Prüfungsordnung durch den Fachbereichsrat gewählte Prüfungsausschuss. ${ }^{22}$ Bisweilen ist Prüfer einer Leistungskontrolle nach den einschlägigen Prüfungsordnungen darüber hinaus der Leiter einer Lehrveranstaltung, ohne dass es eines gesonderten Bestellungsaktes bedarf. ${ }^{23}$ Ausnahmsweise sind schließlich zu Prüfern, auch ohne ausdrückliche Bestellung durch den Ausschuss, satzungsmäßig ${ }^{24}$ berufen die an der Fakultät hauptamtlich tätigen Professoren. ${ }^{25}$

Fest steht damit, dass eine Person, etwa ein Wissenschaftlicher Mitarbeiter, obwohl er gegebenenfalls aufgrund selbstständiger Lehrtätigkeit im Rahmen eines anderweitigen Lehrauftrages die formalen und fachlichen Voraussetzungen hinsichtlich der Prüfungsberechtigung erfüllt, nicht allein dadurch zum Prüfer wird, dass er von einem Professor mit der Korrektur und Bewertung einer bestimmten Leistung betraut wird.

Nichts anderes kann gelten, wenn der derart angewiesene Mitarbeiter nicht vollständig mit der Bewertung beauftragt ist, sondern lediglich eine Vorkorrektur leisten soll, indem er etwa nach dem Modell der nachvollziehenden Korrektur, die Leistung

20 Scholz (Fn. 19), Art. 5 Abs. 3 Rn. 106 f.

21 Vgl. Heckmann/Vogler, Studienbegleitende Zwischenprüfung (Fn. 4), S. 638.

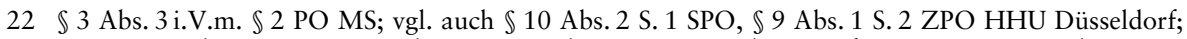
\$21 PO Ruhr-Universität Bochum; $\mathbb{\$} 16$ Abs. 3, 4 PO Köln („Prüfungsamt“); $\mathbb{1 4}$ Abs. 6 S. 1 ZSPO Bucerius Law School; $\mathbb{\$} 17$ Abs. 1 PO Augsburg; $\mathbb{1 4}$ PO Greifswald; $\mathbb{1} 10$ Abs. 3 PO Heidelberg; $\mathbb{} 22$ Abs. 1 S. 3 JuSPO Mannheim; $\mathbb{} 10$ Abs. 1 S. 2 SPO Göttingen.

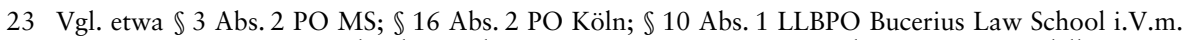
$\$ 7$ ZSPO Bucerius Law School; $\$ 4$ Abs. 1 S. 1 ZPO Göttingen; $\$ 10$ Abs. 2 S. 1 PO Heidelberg.

24 Vgl. BVerwG, Beschl. v. 13.5.1985 - 7 B 54/84; OVG Münster, Urt. v. 23.8.1989 - 1 A 7/87.

25 Vgl. etwa $\$ 10$ Abs. 2 S. 2 SPO HHU Düsseldorf. 
anhand der ihm zugegebenen Lösungsskizze mit einem Bewertungsvorschlag versieht. Bei dem in der juristischen Ausbildung gebräuchlichen Modell der „mittelbaren“ Korrektur fehlt es an der Bewertung durch einen Prüfer überhaupt, wenn die durch gesonderten Bestellungsakt oder kraft Prüfungsordnung zum Prüfer bestellte Person auf eine irgendwie geartete fachwissenschaftliche Vertretbarkeitskontrolle der zu bewertenden Leistung verzichtet. ${ }^{26}$

\section{Normative Grundlagen der Vorkorrektur und ihre Flexion in der Rechtsprechung}

Von der soeben erörterten Problemstellung, wer Prüfer einer universitären Leistungskontrolle sein kann und auf welche Art seine Berufung erfolgt, ist die Frage zu trennen, ob und inwieweit sich ein Prüfer der Unterstützung durch einen Korrekturassistenten bedienen darf. Die Gesetze der Länder sind gegenüber der Vorkorrektur blind. Sie gestalten, wie dargetan, lediglich den allgemein anerkannten Grundsatz „Wer lehrt, prüft“27 aus, verzichten dabei aber auf eine differenzierte Regelung der Modalitäten einer Korrektur. Da das höherrangige Recht wegen seines stärkeren Abstraktionsgrades keine ausdrücklichen Regelungen diesbezüglich enthält, ist die rechtliche Ausgestaltung von Vorkorrekturen damit zunächst in die Hände der Satzungsgeber, der Universitäten und ihrer Fakultäten, gelegt.

Die Grenzen werden im Bereich berufswahlrelevanter Prüfungen maßgeblich durch das Verfassungsrecht, insbesondere den Grundsatz der Chancengleichheit und der Freiheit der Berufswahl (Art. 3 Abs. 1 GG i.V.m. Art. 12 Abs. 1 GG), festgelegt. Widerstreitende Regelungen in Prüfungsordnungen sind, sofern eine verfassungskonforme Auslegung aufgrund ihres eindeutigen Wortlauts ausscheidet, unwirksam. ${ }^{28}$

Inhaltlich regeln die Rechtswissenschaftlichen Fakultäten im Bundesgebiet den Einsatz von Hilfskräften bei der Korrektur schriftlicher Arbeiten in ihren Prüfungsordnungen im Wesentlichen gleich. Im Einzelnen finden sich Formulierungen wie „die Prüfer können bei der Vorbereitung der Bewertung [...] durch [...] wissenschaftliches Personal unterstützt werden“ 29 oder „eine Vorkorrektur kann unter der Verantwortung der Prüferin oder des Prüfers durch eine Korrektorin oder einen Korrektor [...] erfolgen". ${ }^{30}$ Manche Prüfungsordnungen verzichten hingegen ganz auf Ausführungen zu Vorkorrekturen. ${ }^{31}$ Dort, wo Regelungen zur Vorkorrektur vorhanden sind,

26 Vgl. Niehues/Fischer, Prüfungsrecht (Fn. 3), Rn. 320 f. m.w.N.

27 Weber (Fn. 9), $\$ 65$ Rn. 2.

28 Vgl. Niehues/Fischer (Fn. 3), Rn. 320.

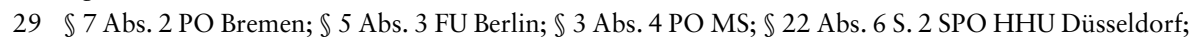

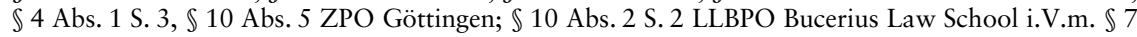
ZSPO Bucerius Law School.

$30 \$ 6$ Abs. 2 PO Köln.

31 Vgl. etwa die Prüfungsordnungen der Universitäten Augsburg, Greifswald, Mannheim und Heidelberg. 
schreiben die Prüfungsordnungen damit das Modell der „nachvollziehenden“ Korrektur ausdrücklich fest.

Im Gleichklang statuiert das Bundesverwaltungsgericht in ständiger Rechtsprechung, dass „[e]in Prüfer [...] die Leistungen des Prüflings persönlich unmittelbar zur Kenntnis zu nehmen und eine selbstständige, eigenverantwortliche, nur seinem Wissen und Gewissen verpflichtete Entscheidung zu fällen" 32 hat, was eben nur bei der „nachvollziehenden“ Korrektur gewährleistet ist. Die „mittelbare“ Korrektur wird damit den Anforderungen der Prüfungsordnungen nicht gerecht. Konkreter urteilt das OVG Münster, der Prüfer könne sich „nicht dadurch aus seiner Verantwortung stehlen, daß er Dritten möglichst enge Vorgaben macht und sie dann an seiner Stelle urteilen und bewerten läßt und sich selbst nur noch die Rolle des Schiedsrichters im Konfliktfall vorbehält “. ${ }^{33}$ In dieser Entscheidung weist das OVG Münster ausdrücklich darauf hin, dass ein Prüfungsverfahren nur dann der persönlichen Bewertungstätigkeit des Prüfers entbehren kann, wenn „die Auswertung der Arbeit und die Zuordnung zu einer Prüfungsnote (...) ohne jede Fachkenntnis von jedermann und eventuell auch von einer Maschine vorgenommen werden kann".34

Bei Korrekturassistenten hingegen bestehen insoweit keine Bedenken an der Rechtmäßigkeit der Regelungen in den Prüfungsordnungen, da selbige schon dem Wortsinne nach lediglich Hilfsarbeiten bei der Bewertung vornehmen und die Unterstützung durch das Hilfspersonal stets im Vorfeld des eigentlichen Bewertungsaktes stattfindet, d.h. die fachwissenschaftliche Richtigkeitskontrolle einer dargestellten Lösung allein in den Händen des Prüfers verbleibt. Denn solange die Beurteilung letztlich durch den Prüfer erfolgt und eine wie auch immer geartete Vorkorrektur nicht das Bewertungsergebnis, sondern nur einen Bewertungsvorschlag darstellt, kommt es nicht zu einer Verkürzung der Rechte eines Prüflings. In diese Richtung geht jedenfalls das dictum des Bundesverwaltungsgerichtes:

„Namentlich kann nicht davon gesprochen werden, dass Korrekturbemerkungen und ähnliche Äußerungen der ersten Prüfer in einer schriftlichen Prüfungsarbeit-anders als etwa in den Fällen der offenen Zweitkorrektur-generell die Besorgnis begründen, dass die zu einer neuen Korrektur berufenen Prüfer auf Grund der möglichen Kenntnis dieser Anmerkungen an einer unvoreingenommenen Prüfung gehindert sind. "35

Vielmehr wird die den Rechten des Prüflings nach zu fordernde und nach dem Grundsatz der Chancengleichheit gebotene Objektivität bei der Bewertung einer

32 BVerwG NJW 2003, S. 1063.

33 OVG Münster NJW 1999, S. 305 (306).

34 OVG Münster NJW 1999, S. 305 (306).

35 BVerwG NJW 2003, S. 1063 (1064). 
Leistung dadurch erweitert, dass der Prüfer bereits die Ansicht eines Dritten kennt, wie die konkrete Leistung zu bewerten ist. Ein verständiger Prüfer, dem die erforderliche Distanz und Unabhängigkeit bei seiner Entscheidungsfindung zuzugestehen ist, wird regelmäßig den Bewertungsvorschlag kritisch hinterfragen und sich bei einer abweichenden Ansicht vertieft mit dem Für und Wider eines Bewertungsergebnisses auseinandersetzen, was letztlich der materiellen Wahrheitsfindung, die den Kern jeder Bewertung ausmacht, besonders zuträglich ist.

Inwieweit dies glaubhaft ist, mag der Leser mit seinem geschulten Judiz selbst beurteilen. Kritisch äußerten sich hierzu bisher etwa Gubl und Pietzcker, wobei Gubl noch vorsichtige Bedenken anbrachte: „Personen, die nicht selbst Prüfer, d.h. nicht Mitglieder der im konkreten Fall tätigen Prüfungsausschüsse sind, darf kein Einfluss auf das Prüfungsergebnis eingeräumt werden. "36 Er konkretisiert weiter, Vorkorrekturen „binden zwar die Prüfer nicht, wirken sich aber zumindest dabingehend aus, dass sie dem Prüfer bestimmte Denkanstöße vermitteln und ihm andere vorenthalten. Es ist nicht auszuschließen, dass eine ausschließlich vom Prüfer selbst vorgenommene Beurteilung anders ausfiele als die vom Außenstehenden vorbereite$t e$ ".37

Pietzcker zweifelte bereits drei Jahre vor Gubl an der Unbeeinflussbarkeit und selbstständigen Urteilsfindung. Anders als bei einer Erstentscheidung bestehe bei der Vorkorrektur das Risiko, dass sich ein Prüfer aufgrund des psychischen Anpassungsdrucks an der Bewertung des Assistenten - und sei es auch nur unbewusst - orientiert. Vor diesem Hintergrund betrachtet Pietzcker die Formel der Rechtsprechung als ein stumpfes Schwert, wenn sie eine Vorkorrektur zulässt, „solange sich der Professor über jede Arbeit ein selbstständiges Urteil bildet und er nicht unbesehen dem Benotungsvorschlag seines Assistenten folgt". ${ }^{38}$ Zudem sei der einem Prüfer zustehende Beurteilungsspielraum Grund dafür, bereits den Prozess der Bewertung möglichst gegen Fehlerquellen abzusichern, weil die damit einhergehende gerichtliche Kontrolldichte gegenüber sonstigen behördlichen Entscheidungen, bei deren Vorbereitung Hilfskräfte mitwirken, deutlich geringer sei. ${ }^{39}$

Pietzckers Zweifel sind angebracht. Korrekturbemerkungen am Rande lenken nicht nur rein physiologisch das Auge des Prüfers auf bestimmte Teile der Klausur, sondern führen auch kognitiv zu einer tieferen Auseinandersetzung; und zwar nicht nur mit der Lösung des Prüflings, sondern gerade auch mit dem Kommentar des Vorkorrektors. Freilich liegt es im Bereich der Möglichkeiten, sich auf den Standpunkt zu stellen, eine identische Klausurlösung müsse ungeachtet jedweder Vorbeeinflussung zu 
einem identischen Bewertungsergebnis führen. Dieser auch im Hinblick auf den Gleichheitsgrundsatz nach Art. 3 Abs. 1 GG wünschenswerte Idealzustand erscheint aber schon bei der Korrektur mehrerer Arbeiten durch einen Prüfer schwerlich erreichbar. Werden die Arbeiten eines Prüfungsdurchganges von mehreren Prüfern korrigiert, ist bereits eine völlige Ergebnisgleichheit in aller Regel nicht mehr zu erzielen. Dies zu fordern, sprengt den Rahmen dessen, was realistischerweise zu leisten ist. Wenngleich Ergebnisidentität durch die Etablierung einer reinen Prüfungsprofessur verwirklicht werden könnte, wäre dies doch mit der Institution der Professur nicht in Einklang zu bringen, wollte man verhindern, dass Prüfungsroboter im Professorenmantel entstehen. Ähnliches gilt für die in jüngerer Zeit zunehmend anzutreffende Honorarprofessur, weil den Angehörigen dieses Personenkreises aufgrund ihrer praktischen Tätigkeit der zeitliche Rahmen nicht zur Verfügung steht, dessen es neben der Lehr- auch für die Prüfertätigkeit bedarf und man auch diesen Personenkreis nicht zu hauptberuflich Prüfenden machen möchte.

Vom universitären Ausbildungskonzept kann daher nur die Gewährleistung eines Verfahrens geboten sein, innerhalb dessen für jede einzelne Bearbeitung die Bewertung durch eine abstrakt fachlich qualifizierte Person sichergestellt ist. In diesem Sinne ist Verfahren Form. Form wiederum ist jedenfalls für das Prüfungsrecht die Zwillingsschwester der Freiheit und die Feindin der Willkür. ${ }^{40}$ Denn Formen „lassen sich nur brechen, nicht biegen. " 41

\section{Korrekturpraxis}

Umreißt das Vorstehende theoretisch die modellabhängige Distanz eines Prüfers zu der Bewertung einer Leistung, kann regelmäßig nicht vorgebracht werden, in der Praxis liefe es anders. Denn berücksichtigt man das im Rahmen einer Veranstaltung anfallende Korrekturaufkommen, insbesondere bei den gut besuchten Veranstaltungen der unteren Semester, ist die Annahme, der jeweilige Veranstaltungsleiter könne die einzelnen Bearbeitungen selbstständig korrigieren und zugleich den an seine Person gestellten Anforderungen auch nur ansatzweise nachkommen, abwegig. Dass dies auch niemand ernstlich in Betracht zieht, belegen zudem die stetigen Hinweise in Schrifttum und Rechtsprechung auf die Unentbehrlichkeit der Unterstützung der Professoren bei der Korrektur „im heutigen Universitätsbetrieb “42 durch Hilfskräfte. Entbehrte die Praxis eines mittelbaren Korrekturverfahrens, wären Ausführungen dieses Inhaltes gegenstandslos.

40 R. von Jhering, Geist des römischen Rechts auf den verschiedenen Stufen seiner Entwicklung, 2. Teil, 2. Abteilung, Aalen 1858, $\mathbb{S} 45$, S. 497; P. Oestmann, Die Zwillingsschwester der Freiheit. Die Form im Recht als Problem der Rechtsgeschichte, in: P. Oestmann (Hrsg.), Zwischen Formstrenge und Billigkeit, Wien 2009, S. 1.

41 v. Jhering, Geist des römischen Rechts (Fn. 41), $\mathbb{S} 45$, S. 497.

42 VGH Bebenhausen, JZ 1959, S. 67; vgl. auch Pietzcker (Fn. 5), S. 118. 
Besonders markant zeigt sich das Verfahren der Juristischen Fakultäten in dem hier aufgezeigten Sinne durch die semesterweise erscheinenden zahlreichen Stellenausschreibungen, die explizit Gesuche von Korrekturpersonal beinhalten. Würde einem Prüfer praktisch stets abverlangt, eine vorkorrigierte Leistung unmittelbar zur Kenntnis zu nehmen und zu bewerten, ist in Anbetracht der finanziellen Situation vieler Hochschulen nicht einsichtig, warum es einer kostspieligen Vorkorrektur bedarf, welche - die einschlägigen Prüfungsordnungen beim Worte genommen - nicht mehr leisten dürfte als eine orthographische Korrektur. Der Gewinn einer derart gestalteten Vorkorrektur wäre in jedem Fall gering und sicher nicht finanziert. Die von einem Prüfer unbesehene Korrektur eines Assistenten hingegen ist keine Korrektur.

Damit steht dem gegenwärtig praktizierten Verfahren dessen Rechtswidrigkeit auf die Stirn geschrieben. Auch wenn gelegentlich die Arbeitsbelastung und Überlastung zum Anlass genommen wird, die gegenwärtige Prüfungspraxis solange zu legitimieren, als eine verbindliche Anleitung an ausreichend sachkundige Korrekturkräfte gewährleistet ist, vermögen diese Umstände keine Rechtfertigung zu liefern, ${ }^{43}$ weil die Kriterien „verbindliche Anleitung“ und „hinreichende Sachkunde“ mangels Konturenschärfe schon im Ansatz versagen müssen. Die formale Rechtswidrigkeit des Prüfungsverfahrens kann zudem ohnehin nicht durch eine verwaltungsfreundliche Auslegung des Prüfungsrechts beseitigt werden. Wie das OVG Münster prägnant statuiert: „Die Konsequenzen, die aus dem Rationalisierungsbedarf bei Prüfungen an einer Massenuniversität zu ziehen sind, kann [...] nur der Normgeber ziehen."44

\section{Reformvorschläge}

Wenn das Verfahren den Anforderungen, welche das Prüfungsrecht an dessen Ausgestaltung stellt, nicht gerecht wird, kann die Lösung schlechterdings sein, dass die Wirklichkeit hinter dem Anspruch zurückbleibt. Genauso wenig können Professoren aber zu den erwähnten „Prüfungsrobotern“ degradiert werden. Ein erfolgversprechender Ansatz dürfte es hier sein, denjenigen Personen auch nach Außen hin die Legitimation zur Prüfungsabnahme einzuräumen, welche den formalen und fachlichen Anforderungen gerecht werden und die in der Sache ohnehin schon befasst sind. Adressat des Formappells muss dann das mit der Prüferbestellung betraute Hochschulorgan sein.

Wenn das Bestellorgan, regelmäßig der Prüfungsausschuss, zusätzlich zu den ohnehin qua Satzung zu Prüfern berufenen Leitern der Lehrveranstaltungen auch die erwähnten Wissenschaftlichen Mitarbeiter mit der Prüfungsabnahme betraut, sollte sich die Korrekturlast der einzelnen Personen auf ein vertretbares Maß reduzieren.

43 M. Sachs, Vorkorrektur schriftlicher Arbeiten in der Schwerpunktbereichsprüfung nach dem neuen JAG, NWVBl. 2004, S. 46 (47).

44 OVG Münster NJW 1999, S. 305 (306). 
Praxis und Theorie versöhnt, dürfte dies allen Beteiligten zum Vorteil gereichen. Nicht nur, weil auf diese Weise die Bereitschaft zur verantwortlichen Auseinandersetzung mit einer zu bewertenden Leistung größer sein sollte, wenn der Korrektor weiß, dass er selbst auch nach außen hin für seine Prüfungsentscheidung einstehen muss, sondern insbesondere weil auch Transparenz des Verfahrens geschaffen wird. Für den Prüfling ist Transparenz Voraussetzung für sein Vertrauen in den Prüfungsapparat, denn „Vertrauen ohne Transparenz, die erlaubt zu verfolgen, was geschieht, ist nicht möglich". ${ }^{45}$

\section{E. Zusammenfassung}

Wie zwiespältig das Verhältnis der Fakultäten zu den Vorgaben des Prüfungsrechtes ist, zeigen die in jeder Prüfungsordnung zu findenden Regelungen, dass die Prüfer von (geeigneten) Korrekturassistenten unterstützt werden können. Vor dem Hintergrund der vorstehenden Diskussion kann solchen Regelungen nicht mehr entnommen werden, als dass ein akutes Legitimationsbedürfnis dafür besteht, Wissenschaftliche Mitarbeiter mit den Bearbeitungen der Prüflinge überhaupt in Kontakt zu bringen. Die Not ergibt sich schlicht daraus, dass die Zuordnung einer Bewertung zu ihrem Prüfer gegenwärtig nicht ganz klar ist. Eine Lösung ist diese: Reformbestrebungen einmal aus der Perspektive des Prüfungsrechtes betrachtet, muss die Form, die Benennung des Prüfers, der Wirklichkeit entsprechen. Der bewertende Mitarbeiter wird zum Prüfer bestellt.

45 BVerfGE 40, 296 (327); vgl. N. Lubmann, Legitimation durch Verfahren, Frankfurt am Main 1983, S. 129. 